\title{
Aetiology, pathogenesis, and pathology of cervical neoplasia
}

\author{
M J Arends, C H Buckley, M Wells
}

\begin{abstract}
Early epidemiological studies of cervical neoplasia suggested a causal relation with sexual activity and human papillomaviruses (HPVs) have emerged as prime suspects as venerally transmitted carcinogens. HPVs fall into two broad camps: low risk types, associated with cervical condylomas and CIN 1; and high risk types (mostly 16 and 18 ), found in $50-80 \%$ of CIN 2 and CIN 3 lesions, and $90 \%$ of cancers. This association with cancer is very strong, with odds ratios of $>15$ (often much higher) in case-control studies that are methodologically sound. An infrequently detected third group of intermediate risk type HPVs is associated with all grades of CIN and occasionally with cancers. HPVs have also been detected in a wide range of asymptomatic controls, indicating that other events are required for development of neoplasia such as viral persistence and/or altered expression of viral genes, often following integration of the viral genome. This leaves the two major viral oncogenes, E6 and E7, directly coupled to viral enhancers and promoters, allowing their continued expression after integration. High risk HPV E7 proteins bind and inactivate the $R b$ protein, whereas E6 proteins bind p53 and direct its rapid degradation. A range of putative

cervical neoplasia, emphasising the role of HPVs.

(F Clin Pathol 1998;51:96-103)

Keywords: human papillomavirus; cervical neoplasia

This review is based on three presentations by the authors to the first National Cervical Screening Conference organised by Marie Curie Cancer Care and held in Oxford, UK in September 1996. It also includes several more recent developments. It is important to emphasise that the cervical screening programme aims to detect women who have an epithelial abnormality that might, if untreated, lead to the development of cervical carcinoma. The screening programme is not designed to detect small or early invasive carcinomas, but no screening system will detect all women at risk and it is inevitable that in a small proportion of smears, evidence of invasive cancer will be found. Cervical smears may also identify nonneoplastic epithelial changes, such as koilocytosis and multinucleation, which are believed to be associated with infection by human papillomavirus (HPV), which is strongly suspected to play an aetiological role in cervical neoplasia. Analysis of the effectiveness of the cervical screening programme and its further development should be based on a clear understanding of the aetiology, pathogenesis, and pathology of cervical neoplasia.
\end{abstract} cofactors has been implicated in progression: HLA type, immunosuppression, sex steroid hormones, and smoking; most of these cofactors appear to influence progression to CIN 3. The natural history includes progression to CIN 3 in $10 \%$ of CIN 1 and $20 \%$ of CIN 2 cases, whereas at least $12 \%$ of CIN 3 cases progress to invasive carcinoma. Cervical glandular intraepithelial neoplasia (CGIN) often coexists with squamous CIN, and the premalignant potential of high grade CGIN is not in doubt, but the natural history of low grade CGIN remains uncertain. A high proportion of CGIN lesions and adenocarcinomas are HPV positive, and HPV18 has been implicated more in glandular than in squamous lesions. A strong clinical case for the application of HPV typing of cells recovered from cervical scrapes can be made; however, a rigorous cost-benefit analysis of introducing HPV typing into the cervical screening programme is required. Prophylactic and therapeutic HPV vaccines are under development. This article reviews the aetiology, pathogenesis, and pathology of
Aetiology of cervical neoplasia

Early epidemiological studies of cervical neoplasia suggested a direct causal relation with sexual activity, measured as multiple sexual partners and early onset of sexual activity. ${ }^{12} \mathrm{~A}$ further risk factor was exposure to the "high risk man", characterised by a history of promiscuous sexual activity and/or sexual exposure to a partner who develops genital neoplasia. Over the past 20 years the search for venereally transmitted carcinogens has included components of semen and various viruses, including Epstein-Barr virus, cytomegalovirus, and herpes simplex II virus (HSV), but without success. The lack of persistent HSV viral DNA, RNA or protein in most neoplastic lesions, and the equivocal findings in prospective studies of $\mathrm{HSV}$ in cervical cancers do not support a key aetiological role for HSV in cervical cancer. ${ }^{3}$ However, HPVs have emerged as prime suspects, being at the scene of the crime carrying the tools to start the job: high risk HPVs are consistently found in over $90 \%$ of cervical cancers and they possess transforming viral oncogenes (E6 and E7). 


\section{Papillomaviruses}

Papillomaviruses are members of the Papovaviridae family with an icosahedral structure composed of 72 capsomers and containing closed, circular, double stranded DNA of approximately $8 \mathrm{~kb}$ in length. They are classified by the host species infected and by the degree of DNA sequence homology within specific viral genes. New HPV types must share less than $90 \%$ identity within the L1 gene compared to existing types. Seventy seven types of HPV have been cloned and identified to date with more in the pipeline. ${ }^{4}$

MORPHOLOGICAL EVIDENCE OF HPV INFECTION IN THE LOWER GENITAL TRACT

The most common manifestation of HPV infection is condyloma acuminata, and in the vast majority of cases this is caused by infection by a non-oncogenic form of HPV, most commonly HPV types $6 \mathrm{~b}$ and 11 . Condylomata acuminata are exophytic, papillary lesions composed of long or short fronds of connective tissue covered by an acanthotic squamous epithelium; the lesions may be multiple. In the cervix and vagina, the epithelium covering the condyloma is usually parakeratotic but may be focally orthokeratotic. Characteristic cellular changes, which are limited to the superficial layers of the epithelium, include individual cell keratinisation, multinucleation, and koilocytotic atypia. Koilocytes are characterised by the presence of perinuclear cytoplasmic vacuolation and nuclear enlargement, hyperchromasia, and irregularity. Similar cytological changes can be seen in a flat, non-papillary epithelium, and in such cases the lesion may be apparent only on colposcopic examination when it may be aceto-white, Schiller positive, and have an abnormal vascular pattern. Such lesions can be distinguished from cervical intraepithelial neoplasia (CIN) by the absence of nuclear atypia in the basal layers of the epithelium.

\section{HUMAN PAPILLOMAVIRUS TYPES AND CERVICAL} NEOPLASIA

Early studies quickly demonstrated that HPVs detected within the cervix fell into two broad camps: low risk types, such as HPV6b and 11 associated with low grade lesions, including cervical condylomas and CIN 1; and high risk types, mostly HPV16 and 18 found in $50-80 \%$ of CIN 2 and CIN 3 lesions, and up to $90 \%$ of invasive cervical cancers. ${ }^{25-9}$ Furthermore, semiquantitative detection methods have shown that high levels of HPV16 DNA have a high predictive value for CIN $2 / 3 .{ }^{10}$ Subsequently, an infrequently detected third group of intermediate risk types, such as HPV 31, 33, and 35, was found to be associated with all grades of CIN and occasionally with cancers. More detailed investigations have detected about $30 \mathrm{HPV}$ types in cervical samples, most of which occur either very infrequently or rarely, including low risk types $40,42,43$, and 44 in low grade lesions, and intermediate types $45,51,52,56,58$, and 59 in all grades of CIN and cancers. ${ }^{11-14}$ However, HPVs have also been detected in a wide range $(3-30 \%)$ of asymptomatic controls, ${ }^{81415}$ indicating that other events are required for development of neoplasia, such as viral persistence and/or altered expression of viral genes. It is clear that only a small minority of persistent HPV infections progress to cancer.

HPV types have different effects on the CIN-cancer sequence. An approximate measure of the risk of transition from precursor to cancer can be calculated using the ratio of HPV type prevalence in squamous cancers to that in dysplastic squamous intraepithelial lesions (cancer:CIN prevalence ratio). Several studies have shown that HPV18 is associated with a higher cancer:CIN ratio than HPV 16, ${ }^{73} 16$ suggesting that HPV 18 confers a more aggressive neoplastic phenotype than HPV16.

EPIDEMIOLOGICAL STRENGTH OF ASSOCIATION OF CERVICAL NEOPLASIA WITH HPV

Data from large, methodologically sound, case-control studies using sensitive and specific polymerase chain reaction (PCR) based assays strongly suggest that HPV infections cause most CIN lesions. ${ }^{8}$ Munoz and Bosch ${ }^{2}$ have recently reviewed the available epidemiological data on the association of HPV and cervical cancer and found that the data fulfil the Bradford Hill criteria of causality; this conclusion was endorsed by an international multidisciplinary group. ${ }^{17}$ The association between cervical cancer and certain HPV types is: (1) very strong, with odds ratios of $>15$ (often much higher) in case-control studies that are methodologically sound; (2) consistent in high risk and low risk countries for cervical cancer; (3) specific for high risk HPV types (HPV16 accounts for the most, followed by HPV18); (4) supported by studies that show HPV infection precedes development of CIN 2/3; and (5) biologically plausible with laboratory evidence of the oncogenic potential of high risk type HPV genes (E6 and E7 viral oncogenes).

Many epidemiological studies have consistently shown that over $90 \%$ of cervical cancers can be attributed to specific HPV types. The small proportion of cancers apparently negative for HPV may be either false negatives, reflecting failure to detect new HPV types or very low levels of known HPV genomes, or true HPV negative cancers and thus comprise a separate group. There is some evidence that HPV negative cancers are found more often in older women and are associated with a poorer prognosis. Furthermore, early studies suggested a possible different molecular pathway of carcinogenesis with p53 mutation found only in HPV negative tumours (see later). ${ }^{18}$

HPV GENOME INTEGRATION AND VIRAL

ONCOGENE EXPRESSION

Important differences in the physical status of HPV DNA have been demonstrated in cervical neoplasms. Low risk HPV types $6 \mathrm{~b}$ and 11 are maintained as extrachromosomal, circular DNA episomes in low grade cervical lesions, whereas the genomes of high risk HPV types 16 and 18 are found integrated into the cellular DNA in most human cervical carcinomas and carcinoma derived cell lines. ${ }^{19}{ }^{20}$ Although the site of viral DNA integration into the host 
genome of carcinomas varies from case to case, the pattern in each cancer is monoclonal suggesting that this occurs before expansion of the malignant clone of cells. There is a consistent pattern of disruption of the circular viral DNA upon integration. The recombination event frequently occurs within the viral genes E1 or E2, sometimes causing focal deletions. ${ }^{192}$ This leaves E6 and E7 directly coupled to the viral enhancer and promoter sequences in the HPV upstream regulatory region, allowing their continued expression after integration. Furthermore, disruption of E1 or E2 during integration inactivates or decouples other early and late viral genes from the viral promoter and enhancer sequences. In particular, this includes E2, which encodes a transcription factor that is the main viral transcription regulator. Full length E2 protein binds to the $\mathrm{E} 2$ recognition sites within the HPV regulatory region adjacent to the viral promoter, and represses viral gene expression. ${ }^{21}$ Loss of E2 function following integration leads to de-repression of the viral promoter, with enhanced expression of E6 and E7 oncoproteins. Thus, HPV DNA integration appears to be a critical event in the progression of cervical neoplasia, as HPV oncogenes E6 and E7 are conserved intact and show evidence of persistent and increased expression in carcinomas. ${ }^{21-23}$ Integration of HPV 16 and 18 DNA also occurs following experimental introduction into human keratinocytes, and this results in the development of a cluster of growth modulation changes including a reduced growth factor requirement (growth in low serum), development of aneuploidy, and immortalisation (rescue from senescence). ${ }^{24}{ }^{25}$ These immortalised keratinocytes may be the equivalent of CIN 2 or CIN 3 in vivo, as raft cultures formed by them show morphological features similar to high grade intraepithelial neoplasia. ${ }^{26}$ All of these changes have been shown to be dependent on expression of high risk HPV oncogenes E6 and E7. ${ }^{26}{ }^{27}$ Introduction of HPV E6 and E7 genes linked to various promoters into transgenic mice, has produced a variety of tumours, the site of which depends on the promoter used, confirming the transforming properties of these viral oncogenes. ${ }^{27}$

E6 and E7 have proved transforming activities in vitro, as E6 and E7 can cooperate with activated ras to transform primary rodent cells and human keratinocytes to malignancy. ${ }^{28-30}$ Their main mechanism of action is reduction of intracellular availability of the host's cell cycle inhibitor (oncosuppressor) proteins p53 and retinoblastoma $(\mathrm{Rb})$ by the E6 and E7 oncoproteins from high risk HPV types 16 and 18 , but not from low risk HPV types $6 \mathrm{~b}$ or 11 . $\mathrm{E} 7$ proteins bind and inactivate the $\mathrm{Rb}$ protein. ${ }^{31-33}$ E6 proteins bind p53 and direct its rapid degradation. ${ }^{343}$ An alternative mode of inactivation of $\mathrm{p} 53$ is by mutation, and this has been shown to be a rare phenomenon in high grade CIN and in HPV positive invasive cervical carcinomas, ${ }^{36}{ }^{37}$ in keeping with loss of p53 function by HPV E6 directed rapid degradation of $\mathrm{p} 53$ protein. However, the early suggestions that p53 mutations are observed only in
HPV negative tumours have not been confirmed by recent studies. ${ }^{38}$ Temporary functional abrogation of $\mathrm{p} 53$ and Rb by HPV E6 and E7 proteins is important for viral DNA replication in senescent or terminally differentiated (non-cycling) squamous epithelial cells. Following viral DNA integration during carcinogenic progression, E6 and E7 are persistently expressed causing profound loss of function of $\mathrm{p} 53$ and $\mathrm{Rb}$ proteins.

These HPV mediated effects on p53 and Rb tend to drive cellular movement around the cell cycle as both p53 and $\mathrm{Rb}$ are negative growth regulators that control transit from $G_{0} / G_{1}$ to $S$ phase. p53 also acts as a "guardian of the genome" and is involved in responding to DNA damage by inducing either growth arrest or cell death by apoptosis. Thus, HPV mediated loss of p53 function decreases the cancer cell's susceptibility to apoptosis, and promotes cellular survival after DNA damage or development of genomic instability, allowing accumulation of genetic changes that may drive further progression to malignancy. ${ }^{18} 3940 \mathrm{HPVs}$ have a third oncogene, E5, which appears to alter signalling from growth factor receptors for epidermal growth factor and platelet derived growth factor. As E5 is often lost or not expressed after viral integration its role in the later stages of cervical carcinogenesis is uncertain, although it may contribute to development of low grade CIN lesions. ${ }^{41}$

CLINICAL APPLICATION OF HPV RESEARCH

There is as yet no clinical situation in which knowledge of the presence of HPV infection or indeed the HPV type, determines clinical management. There is now abundant evidence that women harbouring HPV16 in their cervices are at greater risk of neoplastic progression, and in the presence of negative or mildly abnormal cytology, are more likely also to have a severe histological lesion, such as CIN 3. ${ }^{10243} \mathrm{~A}$ strong clinical case for the application of HPV typing of cells recovered from cervical scrapes can be made. ${ }^{44}$ There are two possible approaches: first, HPV typing could augment or indeed supplant the initial cervical cytological screen; second, HPV typing could be used for cases with borderline or mildly dyskaryotic cervical smears to identify a subgroup at high risk of progression. ${ }^{45}$ However, a rigorous costbenefit analysis of introducing routine HPV typing into the cervical screening programme has yet to be carried out in the UK; some trials are in progress in the Netherlands. Prophylactic and therapeutic HPV vaccines are under development, with some therapeutic vaccines targeted to induce an immune response against tumour cells that express HPV E7 protein. ${ }^{46}$

\section{Pathogenesis and pathology of cervical neoplasia}

COFACTORS AND CERVICAL NEOPLASIA

Epidemiological studies have thrown up a range of putative cofactors involved in progression: HLA type, immunosuppression, sex steroid hormones (based on associations of cervical cancers with high parity and long term use of the oral contraceptive pill), and 
smoking. ${ }^{47-49}$ Furthermore, most of these cofactors appear to influence progression to CIN 3 (from latent HPV infection or low grade CIN), rather than CIN 3 to cancer. ${ }^{2}$ The effects of smoking have been well studied and there is a consistent epidemiological association (odds ratio of about 2) between cigarette smoking and cervical cancer. The carcinogenic constituents of tobacco smoke are strong candidates for co-carcinogenic factors with HPVs in cervical carcinogenesis. Cigarette smokers with mildly abnormal cytology are more likely to have larger and more severe histological lesions. ${ }^{50}$ The constituents of tobacco smoke (such as nitrosamines) can be demonstrated in cervical mucus, and increased smoking related DNA adducts have been shown in normal epithelium next to cervical intraepithelial and invasive neoplasia, indicating a direct effect on cervical epithelium at the DNA level. ${ }^{51}{ }^{52}$ Differential cervical expression of cytochrome P450 enzymes, which activate carcinogenic nitrosamines, and glutathione $S$ transferase, which denatures them, may play a role in determining individual susceptibility to tobacco related carcinogens. ${ }^{53}$

\section{ALTERATIONS IN CELLULAR ADHESION}

MOLECULES

Integrins are transmembrane glycoproteins important in interactions between epithelial cells and basement membranes. Integrin expression is confined to the basal layer of normal squamous epithelium. However, the patterns of integrin expression in neoplastic cervical epithelium differ from this, with dysplastic cells expressing integrins throughout the full thickness of the epithelium in CIN $3 .^{54}$

Epithelial cadherin (E cadherin) is a cell-cell adhesion molecule that connects epithelial cells via homotypic, calcium dependent interactions. In normal cervix, $\mathrm{E}$ cadherin is expressed on the cell membrane of basal and parabasal cells. Cytoplasmic reactivity is present in occasional basal cells only. In CIN, there is increased cytoplasmic reactivity, which is strongly correlated with the grade of the CIN lesion. In squamous carcinomas, reduced membranous and increased cytoplasmic reactivity is seen with worsening differentiation..$^{55}$

\section{CERVICAL INTRAEPITHELIAL NEOPLASIA}

The normal cervix is covered on its outer surface by a non-keratinising, stratified squamous epithelium, which is continuous below with the squamous epithelium lining the vagina, and above abuts onto the mucus secreting columnar epithelium lining the endocervical canal and its associated crypts. The junction between the two epithelia normally coincides with the external os but this is not a constant relation. At puberty, in pregnancy (particularly the first one), and in some steroid contraceptive users, changes in the size and shape of the cervix result in the squamocolumnar junction being carried out on to the anatomical ectocervix. This exposes the tissues previously found in the lower endocervical canal to the vagina. This is a physiological process and the exposed tissue forms the "cervical ectopy".
It is from the epithelium that covers the ectopy that most CINs and invasive carcinomas develop. Under physiological conditions, the columnar epithelium of the ectopy undergoes metaplasia to a stratified squamous epithelium, and it is during this metaplastic process that the epithelium seems to be particularly vulnerable to oncogenic viruses, and perhaps to other factors resulting in the development of an intraepithelial neoplasm rather than a normal epithelium. The intraepithelial neoplasm may be of squamous or columnar cell type.

$\mathrm{CIN}$ is recognised by disturbances of cellular maturation and stratification, and by the presence of cytological atypia. It is customarily graded according to the degree of cytoplasmic maturation ${ }^{56}$ but, by definition, nuclear atypia must be present at all levels of the epithelium. It affects the surface epithelium and extends down into the underlying crypts. In younger women, CIN lies on the ectocervix where the ectopy has formed, but in older women the tissues that form an ectopy are withdrawn into the endocervical canal, therefore, CIN in older women tends to lie within the endocervical canal where it may be more difficult to sample. CIN initially forms a single area and thus, if the resection margins of tissue removed for its treatment are free from disease, it is likely that the whole of the lesion has been removed. ${ }^{56-59}$

\section{Histopathological diagnosis of cervical intraepithelial neoplasia}

In Europe, CIN is usually divided into three grades, although other schemes are used..$^{56}$ The lesions range from a well differentiated intraepithelial neoplasm (CIN 1) to a poorly differentiated intraepithelial neoplasm (CIN 3). CIN 2 occupies an intermediate position. The differentiation of CIN is rarely uniform throughout the affected area, the most poorly differentiated component tending to lie adjacent to the columnar epithelium of the endocervical canal. For a diagnosis of CIN, nuclear abnormalities such as enlargement, pleomorphism, and hyperchromasia must be present at all levels in the epithelium, including the basal layer. Hyperchromasia is not a prerequisite, and in some forms of CIN the nuclei do not stain darkly. The degree of nuclear atypia tends to be greater in CIN 3 than in CIN 1. Normal mitoses vary in number and do not affect the diagnosis, while atypical mitoses may be present in all grades of CIN. In CIN 1, cytoplasmic maturation occurs in the superficial two thirds of the epithelium. In CIN 2 it starts in the middle third of the epithelium, and in CIN 3, cytoplasmic maturation, which is minimal or even absent, occurs only in the most superficial third of the epithelium. Changes usually attributed to the presence of HPV infection, such as koilocytosis and epithelial multinucleation, are often present and are most conspicuous in CIN 1 and 2, and minimal or absent in CIN 3. This may be a reflection of viral integration in the high grade lesions.

Most histopathologists in the UK have not accepted the two tier Bethesda system for 
cervical intraepithelial abnormalities, ${ }^{60}$ but have chosen to retain the present grading system, ${ }^{61}$ together with a recently introduced category of epithelial abnormality to encompass lesions in which a diagnosis of CIN cannot be made with certainty: basal abnormality of uncertain significance. ${ }^{56}$ This category may be particularly useful in combating the problem of overdiagnosis of CIN 1, still thought to occur to a considerable extent, because pathologists fail to appreciate that, even in a CIN 1 lesion, the nuclei are abnormal throughout the full thickness of the epithelium and not just in the lower third above which there is cytoplasmic maturation. However, it is possible to distinguish between CIN 1 and HPV infection alone by virtue of the presence of koilocytosis without nuclear atypia.

\section{Natural history of cervical intraepithelial neoplasia}

Review of the world literature ${ }^{62}$ on the natural history of CIN indicates that the approximate likelihood of regression of CIN 1 is $60 \%$, persistence $30 \%$, progression to CIN $310 \%$, and progression to invasion $1 \%$. The corresponding approximations for CIN 2 are $40 \%, 40 \%$, $20 \%$, and $5 \%$, respectively. The likelihood of CIN 3 regressing is $33 \%$ and progressing to invasion greater that $12 \%$. Thus, even the higher degrees of atypia may regress in a significant proportion of cases, and although the probability of an atypical epithelium becoming invasive increases with the severity of the atypia, this does not occur in every case.

As $50-60 \%$ of the lesions diagnosed as CIN 1 will regress spontaneously, if clinicians intervene at such an early phase of the process it may result in the unnecessary treatment of women who are at little or no risk of developing carcinoma. A balance has to be struck, however, between unnecessary treatment and a failure to eradicate a lesion that has the potential to progress to invasive carcinoma. In general, persistence of CIN 1 is regarded as an indication for eradication. It is also recognised that higher grade lesions of CIN 2 and 3 are more likely to progress to invasive carcinoma and are usually treated without undue delay.

\section{Invasive potential of CIN 3}

No one doubts the malignant potential of high grade CIN since the debacle at the National Women's Hospital in Auckland, New Zealand in the 1970 s, in which many women with persistent abnormal cytology following biopsy of "carcinoma in situ" (as it was called at that time) were treated conservatively. Twenty nine of 131 women $(22 \%)$ developed invasive cervical cancer (compared to $1.5 \%$ of a control group), and eight died. ${ }^{63}$

The main histological features identified in CIN 3 associated with microinvasive carcinoma or predictive of subsequent microinvasion are extensive involvement of surface epithelium and deep endocervical crypts by expansile CIN 3, luminal necrosis, and intraepithelial squamous maturation. ${ }^{64}$ Other features more commonly present in CIN 3 associated with microinvasive carcinoma in- clude frequent mitosis and apoptosis, pericryptal concentric fibroplasia, pericryptal inflammatory infiltrates, pronounced cellular pleomorphism, certain nuclear changes (distinct nucleoli and chromatin clearing), and the emergence of streams of darkly staining spindle cells orientated at right angles to the basement membrane. In a prospective study, $83 \%$ of cases illustrating the major microinvasive carcinoma associated features revealed evidence of microinvasive carcinoma or frank invasion either on serial sections of the original biopsy or on subsequent biopsy. None of the 64 cases of CIN 3 that lacked these features showed evidence of invasion on serial sections or on follow up over 18 months. When these features are present in a biopsy specimen, serial sections should be performed to exclude the presence of microinvasion, and closer clinical follow up of these patients is appropriate.

INVASIVE CARCINOMA OF THE CERVIX

Most invasive carcinomas of the cervix develop from an intraepithelial neoplasm that has formed in the tissues of the cervical ectopy. ${ }^{56} 57$ They tend to lie, therefore, on the ectocervix in younger women and in the endocervical canal in older woman (see above). The tumours may have an exophytic or endophytic pattern of growth, those on the ectocervix being more commonly exophytic and those in the endocervical canal more commonly endophytic. This has clinical importance in that exophytic tumours on the ectocervix are less likely to have extended into the adjacent tissues and organs than endophytic tumours of similar size, and are less likely to have metastasised..$^{58}$

Cervical carcinomas spread locally into the cervical stroma, the paracervical and parametrial tissues, the body of the uterus, the vagina, and, late in the course of the disease, to the bladder and rectum. ${ }^{56-59}$ Lymphatic permeation in the cervical stroma may occur even when the tumour is of low volume, therefore, metastases may be found in the pelvic lymph nodes even when the tumour is small. It is not possible, therefore, to equate "small" with "early" carcinoma.

MICROINVASIVE SQUAMOUS CARCINOMA OF THE CERVIX

It is customary to stage invasive carcinomas of the cervix according to the criteria laid down by FIGO. ${ }^{596566}$ The FIGO guidelines do not use the term microinvasive carcinoma, but stage $1 \mathrm{~A}$, which encompasses lesions that can be diagnosed only on microscopy, and are usually regarded as being synonymous with microinvasive carcinoma. When invasive disease is identified unexpectedly in women who have been regularly screened, the carcinomas often come into this category. Such a finding should not be regarded as a failure of the screening process because such neoplasms carry only a low risk of metastatic disease and can, therefore, usually be treated relatively conservatively.

FIGO has recently modified its classification of microinvasive carcinoma by subdividing stage 1A. Stage 1 (carcinoma strictly confined 
to the cervix) is divided into stage 1A (invasive cancer only identified histologically) and stage 1B (gross lesions even those with only superficial invasion). Stage $1 \mathrm{~A}$ is further divided into stage $1 \mathrm{~A} 1$ (invasion of stroma up to $3 \mathrm{~mm}$ in depth and no wider than $7 \mathrm{~mm}$ ) and stage $1 \mathrm{~A} 2$ (invasion of stroma of 3-5 $\mathrm{mm}$ in depth and no wider than $7 \mathrm{~mm}$ ). There have only been three cases of nodal metastases in 1543 patients in the literature whose microinvasive carcinomas invaded the stroma to $1 \mathrm{~mm}$ or less (termed early stromal invasion). Therefore it appears that early stromal invasion behaves in the same manner as high grade CIN and may be treated by conisation alone provided the cone has been adequately sectioned, the margins are free from tumour, and the curettings do not contain abnormal epithelium. For microinvasive carcinomas invading between 1 and $3 \mathrm{~mm}$, nodal involvement was found in only five of 809 $(0.6 \%)$ patients who had pelvic lymph node dissection, and the vast majority of these cases can also be safely treated by conisation alone. For tumours penetrating $3-5 \mathrm{~mm}$ (FIGO stage 1A2), there appears to be a higher risk of recurrence and nodal metastasis; nodal involvement has been reported in 14 of 214 patients $(6.5 \%){ }^{67}$

The introduction of the revised staging system has recently been criticised. ${ }^{68}$ The term early stromal invasion (up to $1 \mathrm{~mm}$ invasion) is missing in the new version of the staging system and has been replaced by "microinvasion changes" with a depth of invasion up to $3 \mathrm{~mm}$. This group will inevitably include a large number of cases of early stromal invasion $(<1 \mathrm{~mm})$. Burghardt et al reviewed cases in the literature with invasion of $1-3 \mathrm{~mm}$ (cases with early stromal invasion $(<1 \mathrm{~mm})$ were excluded) and found that $1.4 \%$ of patients died of disease. ${ }^{68}$ That is six times more than the death rate of patients with early stromal invasion $(<1 \mathrm{~mm})$ and not significantly different from the proportion of patients $(1.7 \%)$ dying of cancer whose neoplasms extend to a depth of between 3 and $5 \mathrm{~mm}$ at diagnosis. These authors feel, therefore, that it is inappropriate to dilute the stage 1A1 group with a large number of cases in an extremely low risk category (those showing early stromal invasion of $<1 \mathrm{~mm}$ ), which makes treatment results highly favourable. They consider that only early stromal invasion $(<1 \mathrm{~mm})$ merits recognition as a separate category.

There is no convincing evidence in the literature that capillary-like space involvement is associated with lymph node metastases or an increased incidence of recurrence. Therefore, permeation of endothelial lined spaces is ignored for the purposes of staging.

The term microinvasive carcinoma can be applied to squamous carcinomas, adenosquamous carcinomas, and adenocarcinomas (see below). It cannot, however, be used for small cell (neuroendocrine) tumours to which the term microinvasive carcinoma should not be applied even when the tumour is small enough to be stage 1A. Stromal invasion from both a squamous and columnar cell intraepithelial neoplasm is recognised by loosening of the stroma at the point of invasion, and the presence of a variable stromal round cell infiltrate. In the epithelium, at the point of invasion, there is often a subtle change in the pattern of tumour cell differentiation with increased eosinophilia of the cytoplasm and a change in the degree of nuclear atypia. It is important for clinical management that the depth of invasion is measured accurately for microinvasive carcinomas, taken from the base of the epithelium (either surface or glandular) from which it originates.

WHAT IS AN EARLY CARCINOMA OF THE CERVIX? There is some agreement that stage $1 \mathrm{~A}$ carcinomas can be described as "early" although this may be a simplistic view of lesions that may be of limited invasive capacity rather than early. Tumours that are too large to be encompassed by the definition of stage $1 \mathrm{~A}$ but are still confined to the cervix are included in stage $1 \mathrm{~B}$. For tumours of stage $1 \mathrm{~B}$ and higher, radical treatment is required and it would, in our opinion, be inappropriate to include such lesions in a description of early cervical tumours, although it could be argued that the term early carcinoma could include all invasive lesions for which surgery is the first line of treatment.

It has been customary to regard stages $1 \mathrm{~A}$, $1 \mathrm{~B}$, and $2 \mathrm{~A}$ carcinomas as early lesions; however, as metastatic disease may already be present in women with stages $1 \mathrm{~A} 2,1 \mathrm{~B}$, and $2 \mathrm{~A}$ disease it is clearly inappropriate to call these tumours early. Moreover, the pathologist is rarely in a position to identify, with any certainty, the woman at risk of having metastatic carcinoma from the appearance of the initial cervical biopsy in which the invasion is recognised. It is more important to consider not just the size of the lesion at the time of diagnosis, which is the result of a balance between tumour cell gain and loss, ${ }^{69}$ but also to consider the biological aggressiveness of the invasive lesion and, to this end, studies of oncogene activation and overexpression, and disturbances of tumour suppressor genes have been done. ${ }^{70}$ It is apparent from these studies that small volume tumours may behave in quite different ways and many are, in apparent contradiction to their small size, actually advanced carcinomas. A more detailed description of the pathology of advanced, established carcinoma of the cervix can be found elsewhere..$^{57-59} 71$

\section{CERVICAL GLANDULAR INTRAEPITHELIAL}

NEOPLASIA

The term cervical glandular intraepithelial neoplasia (CGIN) is now used to encompass adenocarcinoma in situ and glandular atypia in the endocervix. CGIN is much less common than CIN but, as with CIN, most CGIN lesions occur in the region of the cervical ectopy. In contrast to CIN, in about $10 \%$ of CGIN cases, separate foci may be found higher in the endocervical canal. ${ }^{72}$ Both the surface epithelium and the underlying crypts may be affected by the process, and the junction between the atypical columnar epithelium and the normal columnar epithelium is invariably abrupt. In typical CGIN lesions there are 
disturbances of both crypt architecture and columnar cell differentiation. Crypts become more complex in shape with outpouchings and infoldings of the lining, and they come to lie closer together than usual. The glandular epithelium is stratified, there is a reduction in intracytoplasmic mucin, and an increase in nucleo:cytoplasmic ratios. The nuclei are hyperchromatic and pleomorphic to a variable degree. Nucleoli may be large and multiple. Mitoses are frequent and atypical forms may be present. The columnar cells may remain recognisably endocervical, but intestinal metaplasia is not uncommon. The criteria for grading CGIN are not fully established or agreed, therefore, it is suggested that until the grading system is further evaluated, the range of CGIN lesions be termed either high grade CGIN, corresponding to adenocarcinoma in situ, or low grade CGIN for lesser degrees of abnormality. ${ }^{71} 73$ The premalignant potential of high grade CGIN (adenocarcinoma in situ) is not in doubt, but the true natural history of low grade CGIN remains uncertain. CGIN often co-exists with squamous $\mathrm{CIN}^{74}$ and for this reason may sometimes be overlooked. Abnormalities of oncoprotein expression have been demonstrated in CGIN. ${ }^{71}$

The association between CGIN and HPVs is less clear cut than for squamous lesions, but a high proportion of adenocarcinomas are HPV positive using the sensitive polymerase chain reaction technique. ${ }^{75}$ Some workers have suggested that HPV type 18 is implicated more in adenocarcinoma than in squamous lesions. ${ }^{71}$

ADENOCARCINOMA OF THE CERVIX

Invasive adenocarcinoma is reported to be increasing in incidence, particularly in young women, and there have been suggestions of a link between cervical adenocarcinoma and the contraceptive pill, particularly with prolonged use. $^{76}$ The practical assessment of microinvasive adenocarcinoma of the cervix is much more difficult than its squamous counterpart, because recognition of the point of invasion and therefore its measurement is associated with problems of definition. However, recent work confirms that lesions with a volume of less than $500 \mathrm{~mm}^{3}$ have a negligible risk of local recurrence and lymph node metastases. ${ }^{77}$ It is probably not practical on a day to day basis to express invasion in three dimensions, and gynaecologists tend to be less conservative in their treatment of microinvasive adenocarcinomas arising in the endocervix than microinvasive squamous cervical carcinomas.

The authors thank Dr Euphemia McGoogan, who chaired the session on "Aetiology, pathogenesis and pathology of cervical neoplasia" at the National Cervical Screening Conference, Oxford, September 1996, for encouraging the authors to write this review based on their presentations at that session, and for this review based on their presentations at that session, and for
critical appraisal of the manuscript. Thanks are also due the critical appraisal of the manuscript. Thanks are also due the
NHS Cervical Screening Programme, including Mrs Julietta Patnick as National Co-ordinator, and to Marie Curie Cancer Care for organising and funding the conference.

1 Munoz N, Bosch X, Kaldor JM. Does human papillomavirus cause cervical cancer? The state of the epidemiological rus cause cervical cancer? The Br $\mathcal{f}$ Cancer 1987;57:1-5.

2 Munoz N, Bosch FX. Current views on the epidemiology of HPV and cervical cancer. In: Lacey C, ed. Papillomavirus reviews: current research on papillomaviruses. Leeds: Leeds reviews: current research on papillo
University Press, 1996:227-37.
3 Vonka V, Kanka J, Roth Z. Herpes simplex type II and cervical neoplasia. Adv Cancer Res 1987;48:149-91.

4 Van Ranst M, Tachezy R, Burk RD. Human papillomaviruses: a never ending story? In: Lacey C. Papilomavirus reviezws: current research on papillomaviruses. Leeds: Leeds University Press, 1996:1-19.

5 Arends MJ, Wyllie AH, Bird CC. Papillomaviruses and human cancer. Hum Pathol 1990;21:686-98.

6 Arends MJ, Donaldson YK, Duvall E, et al. HPV in full thickness cervical biopsies: high prevalence in CIN 2 and CIN 3 detected by a sensitive PCR method. F Pathol 1991; 165:301-9.

7 Arends MJ, Donaldson YK, Duvall E, et al. HPV 18 associates with more advanced cervical neoplasia than HPV 16. Hum Pathol 1993;24:432-7.

8 Schiffman MH, Bauer HM, Hoover RN, et al. Epidemiologic evidence showing that human papillomavirus infection causes most cervical intraepithelial neoplasia. 7 Natl Cancer Inst 1993;85:958-64.

9 Schiffman MH, Bauer HM, Lorincz AT, et al. Comparison of southern blot hybridisation and polymerase chain reaction methods for the detection of human papillomavirus DNA. F Clin Microbiol 1991;29:573-7.

10 Cuzick J, Terry G, Ho L, et al. Type-specific human papillomavirus DNA in abnormal smears as a predictor of high grade cervical intraepithelial neoplasia. $\mathrm{Br} f \mathrm{f}$ Cancer 1994;69:167-71.

11 deVilliers E-M, Wagner D, Schneider A, et al. Human papillomavirus infections in women with and without abnormal cervical cytology. Lancet 1987;ii:703-5.

12 Stanley M. Genital papillomaviruses, polymerase chain reaction and cervical cancer. Genitourin Med 1990;66:41517 .

13 Lorincz AT, Reid R, Jenson AB, et al. Human papillomavirus infection of the cervix: relative risk associations of 15 rus infection of the cervix: relative risk associations of 15 37.

14 Van den Brule AJC, Snijders PJF, Meijer CJLM, et al. PCR based detection of genital HPV genotypes: an update and future perspectives. In: Lacey C, ed. Papillomavirus reviews: current research on papillomaviruses. Leeds: Leeds University Press, 1996:181-8.

15 Guerrero E, Shah KV. Polymerase chain reaction in HPV diagnosis. In: Lacey C, ed. Papillomavirus reviews: current research on papillomaviruses. Leeds: Leeds University Press, 1996:175-80.

16 Kurman RJ, Schiffman MH, Lancaster WD, et al. Analysis of individual papillomavirus types in cervical neoplasia: a possible role for type 18 in rapid progression. Am $\mathcal{F}$ Obstet Gynecol 1988;159:293-6.

17 International Agency for Research on Cancer (IARC). Human papillomaviruses. Monographs on the evaluation of the carcinogenic risk of chemicals to humans. Vol. 64 Lyon: IARC, carcino 1995.

18 Crook T, Vousden KH. HPV oncoprotein function. In: Lacey C, ed. Papillomavirus reviews: current research on papillomaviruses. Leeds: Leeds University Press, 1996:55-60.

19 Schwarz E, Freese UK, Gissmann L, et al. Structure and transcription of human papillomavirus sequences in cervical carcinoma cells. Nature 1985;314:111-14.

20 Choo K-B, Pan C-C, Han S-H. Integration of human papillomavirus type 16 into cellular DNA of cervical carcinoma: preferential deletion of the E2 gene and invariable retention of the long control region and the E6/E7 open reading frames. Virology 1987;161:259-61.

21 Thierry F. HPV proteins in the control of HPV transcription. In: Lacey C, ed. Papillomavirus reviews: current research on papillomaviruses. Leeds: Leeds University Press, 1996: 21-9.

22 Shirasawa H, Tomita Y, Sekiya S, et al. Integration and transcription of human papillomavirus type 16 and 18 sequences in cell lines derived from cervical carcinomas. $\mathcal{F}$ Gen Virol 1987;68:583-91.

23 Smotkin D, Wettstein FO. The major human papillomavirus protein in cervical cancers is a cytoplasmic phosphoprotein. F Virol 1987;61:1686-9.

24 Woodworth CD, Bowden PE, Doniger J, et al. Characterization of normal human exocervical epithelial cells immortalized in vitro by papillomavirus types 16 and 18 DNA. Cancer Res 1988;48:4620-8.

25 Woodworth CD, Doniger J, DiPaolo JA. Immortalization of human foreskin keratinocytes by various human papillomavirus DNAs corresponds to their association with cervical virus DNAs corresponds to their as

26 McDougall JK. HPV immortalisation and transformation. In: Lacey C, ed. Papillomavirus reviews: current research on 53 .

27 zur Hausen H. Molecular pathogenesis of cancer of the cervix and its causation by specific human papillomavirus types. Curr Top Microbiol Immunol 1994;186:131-56.

28 Storey A, Pim D, Murray A, et al. Comparison of the in vitro transforming activities of human papillomavirus types. EMBO F 1988;7:1815-20.

29 Durst M, Gallahan D, Jay G, et al. Glucocorticoid enhanced neoplastic transformation of human keratinocytes by human papillomavirus type 16 and an activated ras oncogene. Virology 1989;173:767-71.

30 Arends MJ, McGregor AH, Wyllie AH. Apoptosis is inversely related to necrosis and determines net growth in tumours bearing constitutively expressed myc, ras and HPV oncogenes. Am f Pathol 1994;144:1045-57.

31 Phelps WC, Yee CL, Munger K, et al. The human papillomavirus type $16 \mathrm{E} 7$ gene encodes transactivation 
and transformation functions similar to those of adenovirus E1A. Cell 1988;53:539-47.

32 Munger $\mathrm{K}$, Werness BA, Dys of human papillomavirus E7 proteins with the retinoblastoma tumor suppressor gene product. EMBO $\mathcal{f} 1989 ; 8$
4099-105.

33 Munger K, Yee CL, Phelps WC, et al. Biochemical and biological differences between E7 oncoproteins of the high and low risk human papillomavirus types are determined by amino terminal sequences. $\mathcal{F}$ Virol $1991 ; 65: 3943-8$.

34 Werness BA, Levine AJ, Howley PM. Association of human papillomavirus types 16 and 18 proteins with p53. Science 1990;248:76-9

35 Scheffner M, Munger K, Byrne JC, et al. The state of the p53 and retinoblastoma genes in human cervical carcinoma cell lines. Proc Natl Acad Sci USA 1991;88:5523-7.

36 Lane SA, Wells M. Human papillomavirus, p53 and cervical neoplasia. F Pathol 1994;172:299-300.

37 Jeffers MD, Richmond J, Farquharson M, et al. p53 immunoreactivity in cervical intraepithelial neoplasia and nonnoreactivity in cervical intraepithelial neoplasia and non1994;47:1073-76.

38 Busby-Earle RMC, Steel CM, Williams ARW, et al. p53 mutations in cervical carcinogenesis: low frequency and lack of correlation with human papillomavirus status. $\mathrm{Br} \mathcal{F}$ Cancer 1994;69:732-7.

39 White AF, Livanos EM, Tlsty T. Differential disruption of genomic integrity and cell cycle regulation in normal human fibroblasts by the HPV oncoproteins. Genes Develop 1994;8:666-77

40 Arends MJ, Wyllie AH, Bird CC. HPV 18 is associated with less apoptosis in fibroblast tumours than HPV 16. Br $f$ Cancer 1995;72:646-9.

41 Banks L, Matlashewski G. Biochemical and biological activities of the HPV E5 proteins. In: Lacey C, ed. Papillomavirus reviews: current research on papillomaviruses. Leeds: mavirus reviews: current research on papi

42 Garrenstroom KN, Melkert P, Walboomers JMM, et al. Human papillomavirus DNA and genotypes: prognostic Human papillomavirus DNA and genotypes: prognostic
factors for progression of cervical intraepithelial neoplasia. factors for progression of cervical in

43 Herrington CS, Evans MF, Hallam NF, et al. Human papillomavirus status in the prediction of high grade cervical intraepithelial neoplasia in patients with persistent lowgrade cytological abnormalities. Br f Cancer 1995;71:2069 .

44 Cox JT. HPV DNA testing: clinical boon or boondogle? Lancet 1995;346:717-18.

45 Londesborough P, Ho L, Terry G, et al. Human papillomavirus genotype as a predictor of persistence and development of high-grade lesions in women with minor cervical abnormalities. Int 7 Cancer 1996;69:364-8.

46 Brandsma JL. Animal models for human papillomavirus vaccine development. In: Lacey C, ed. Papillomavirus reviews: current research on papillomaviruses. Leeds: Leeds reviews: current research on papilh
University Press, 1996:69-78.

47 Khare S, Tang S-C, Pater MM, et al. Cofactors with HPV in infection and oncogenesis. In: Lacey C, ed. Papillomavirus infection and oncogenesis. In: Lacey C, ed. Papillomavirus reviews: current research on papillo

48 Benton EC, Arends MJ. Human papillomavirus in the immunosuppressed. In: Lacey C, ed. Papillomavirus reviews: current research on papillomaviruses. Leeds: Leeds University Press, 1996:271-9.

49 Arends MJ, Benton EC, McLaren KM, et al. Renal allograft recipients with high susceptibility to cutaneous malignancy have an increased prevalence of human papillomavirus DNA in skin tumours and a greater risk of anogenital malignancy. Br f Cancer 1997;75:722-8.

50 Luesley D, Blomfield P, Dunn J, et al. Cigarette smoking and histological outcome in women with mildly dyskaryotic cervical smears. Br F Obstet Gynaecol 1994;101:49-52.

51 Sasson IM, Haley NJ, Hoffmann D, et al. Cigarette smoking and neoplasia of the uterine cervix: smoke constituents in cervical mucus. $N$ Engl f Med 1985;312:315

52 Ali S, Astley SB, Sheldon TA, et al. Detection and measurement of DNA adducts in the cervix of smokers and measurement of DNA adducts in the cervix of smo

53 Warwick AP, Redman CWE, Jones PW, et al. Progression of cervical intraepithelial neoplasia to cervical cancer: cytochrome P 450 CYP2D6 EM and glutathione S-transferase GSTM1 null genotypes and cigarette smoking. Br f Cancer 1994;70:704-8.
54 Hughes DE, Rebello G, Al-Nafussi A. Integrin expression in squamous neoplasia of the cervix. F Pathol 1994;173:97104.

55 Vessey CJ, Wilding J, Folarin N, et al. Altered expression and function of E-cadherin in cervical intraepithelial neoplasia and invasive squamous cell carcinoma. F Pathol 1995;176: $151-9$.

56 Anderson MC, Brown C, Buckley $\mathrm{CH}$, et al. Current views on cervical intraepithelial neoplasia. $\mathcal{F}$ Clin Pathol 1991;44: 969-78.

57 Anderson MC. Premalignant and malignant squamous lesions of the cervix. In: Fox $\mathrm{H}$, ed. Haines and Taylor: obstetrical and gynaecological pathology. Edinburgh: Churchill Livingstone, 1995:273-322.

58 Buckley CH. Tumors of the cervix uteri. In: Fletcher CDM, ed. Diagnostic histopathology of tumors. Edinburgh: Churchill Livingstone, 1995b:478-94.

59 Lowe DG, Buckley CH, Fox H. Advances in gynaecological pathology. In: Anthony PP, MacSween RNM, Lowe DG, eds. Recent advances in histopathology. Edinburgh: Churchill Livingstone, 1997:113-37.

60 National Cancer Institute Workshop. The 1988 Bethesda system for reporting cervical/vaginal cytologic diagnoses. Hum Pathol 1988;21:704-8.

61 Richart RM. Natural history of cervical intraepithelial neoplasia. Clin Obstet Gynecol 1968;10:748-84.

62 Ostör AG. Natural history of cervical intraepithelial neoplasia: a critical review. Int $\mathcal{F}$ Gynecol Pathol 1993;12: 186-92.

63 McIndoe WA, McLean MR, Jones RW, et al. The invasive potential of carcinoma in situ of the cervix. Obstet Gynecol 1984;64:451-8.

64 Al-Nafussi Al, Hughes DE. Histological features of CIN 3 and their value in predicting invasive microinvasive squamous carcinoma. f Clin Pathol 1994;47:799-804.

65 Shepherd JH. Revised FIGO staging for gynaecological cancer. Br F Obstet Gynaecol 1989;96:889-92.

66 Shepherd JH. Staging announcement. FIGO staging of gynaecologic cancers: cervical and vulval [erratum Int $\mathcal{f}$ Gynecol Cancer 1995;5:465]. Int F Gynecol Cancer 1995;5: 319.

67 Östör AG, Rome RM. Micro invasive squamous cell carcinoma of the cervix: a clinico-pathologic study of 200 cases with long-term follow-up. Int f Gynecol Cancer 1994; 4:257-4.

68 Burghardt E, Östor A, Fox H. The new FIGO definition of cervical cancer stage 1A: a critique. Gynecol Oncol 1997;65: $1-5$.

69 Arends MJ. How do cancer cells die? Apoptosis and its role in neoplastic progression. In: Leake R, Gore M, Ward RH, eds. The biology of gynaecological cancer. London: RCOG Press, 1995:73-91.

70 Buckley CH. Oncogene expression in gynaecological cancer. In: Leake R, Gore M, Ward RH, eds. The biology of gynaecological cancer. RCOG Press, 1995a:21-33.

71 Griffin NR, Wells $M$. Pre-malignant and malignant disease (adenocarcinoma) of the endocervix. In: Fox H, ed. Haines \& Taylor: obstetrical and gynaecological pathology. 4th ed. Edinburgh: Churchill Livingstone, 1995:323-43.

72 Östör AG, Pagano R, Davoren RA, et al. Adenocarcinoma in situ of the cervix. Int f Gynecol Pathol 1984;3:179-90.

73 NHS CSP. The reporting of histological specimens in the context of the cervical screening programme. Sheffield: NHS CSP Publications. [In press.]

74 Casper GR, Ostor AG, Quinn MA. A clinicopathologic study of glandular dysplasia of the cervix. Gynecol Oncol 1997;64:166-70.

75 Duggan MA, Benoit JL, McGregor SE, et al. Adenocarcinoma in situ of the endocervix: human papillomavirus determination by dot blot hybridization and polymerase chain reaction amplification. Int f Gynecol Pathol 1994;13: 143-9.

76 Ursin G, Peters RK, Henderson BE, et al. Oral contraceptive use and adenocarcinoma of the cervix. Lancet 1994;344:1390-4.

77 Kasper HG, Dinh TV, Doherty MG, et al. Clinical implications of tumor volume measurement in stage 1 adenocarcinoma of the cervix. Obstet Gynecol 1993;81:296-300. 